\title{
La dynamique de la syllepse dans la construction fictionnelle chez Boris Vian
}

Résumé : Cet article présente une étude de la syllepse, figure du double sens, dans un corpus constitué des œuvres romanesques de Boris Vian. Il vise à étudier le fonctionnement des jeux de mots reposant sur une syllepse dans la construction fictionnelle. L'étude précise de la figure permet ainsi d'interroger son rendement littéraire. En commençant par étudier la part de la syllepse dans les jeux de mots (et en la mettant en perspective par rapport au calembour), cet article s'attache au fonctionnement du jeu de mots par syllepse ainsi que la part de la figure dans le défigement. Il s'avère que Vian crée un rapport privilégié entre syllepse et déconstruction des normes langagières. Cette étude s’intéresse ensuite à l'hétérogénéité énonciative à l'œuvre dans les syllepses. Du fait de la cohabitation de deux isotopies, ou de deux acceptions d'un même terme, la syllepse est un lieu de rencontre de points de vue, portant chacun une représentation différente du monde. Enfin, l'article élabore une pragmatique de la syllepse dans un corpus vianesque. La figure, en tant que support du jeu de mots, entre dans la construction d'un monde fictionnel particulier. Elle crée de nouvelles normes tout en étant normalisée par le contexte. En ce sens, elle permet de négocier la fiction auprès du lecteur. La syllepse est alors un outil particulièrement efficace de la fictionnalisation puisque, tout en proposant de nouvelles normes, elle crée un lien particulier avec le lecteur afin de le faire adhérer aux nouvelles règles proposées.

Mots clés : Boris Vian, défigement, énonciation, fictionnalisation, hétérogénéité énonciative, jeu de mots, littérature, pragmatique, syllepse

\section{Introduction}

Boris Vian n'a été reconnu en tant qu'écrivain que de manière posthume. Ses romans ont été publiés entre 1946 et 1953, mais il faut attendre les années 60 pour que se développe l'engouement du public. Vian joue ; l'écrit est pour lui un terrain fertile pour le déchaînement des mots. Il me semble qu'à trop percevoir le jeu et l'amusement, on peut tomber dans la même dérive que les contemporains de Vian : restreindre cet auteur éclectique au récréatif. Vian joue certes avec la langue, mais son rapport à celle-ci dépasse le simple jeu et il est nécessaire de 
prendre en compte sa dimension fictionnalisante - c'est-à-dire son apport dans la construction de la fiction. Le corpus choisi pour cette étude a été établi à partir de l'ensemble des œuvres romanesques signées Boris Vian (Troubles dans les andains, Vercoquin et le plancton, L'Écume des jours, L'automne à Pékin, L'Herbe rouge et L'Arrache cœur). ${ }^{1}$ À partir de ce premier ensemble, un relevé systématique des jeux de mots a révélé l'importance de la syllepse dans la construction de jeux de mots. Sa prégnance dans l'œuvre et son influence dans l'imaginaire vianesque en font un stylème de l'œuvre de Boris Vian.

Les jeux de mots, malgré les nombreuses études les prenant pour sujet (Guiraud 1979 ; Winter-Froemel et Zirker 2015a, 2015b ; Zirker et Winter-Froemel 2015), continuent de poser des questions. Michelle Lecolle (2015 : 217) envisage les jeux de mots «comme une pratique ludique ou poétique délibérée et consciente mettant en relation des mots, et comme une manifestation du sentiment linguistique - conception et perception, non explicitée, de la langue». Laélia Véron (2015 : 94) les considère quant à elle comme la « manifestation d'une liberté créative ludique [...]. Faire un jeu de mots c'est proposer un énoncé nouveau, surprenant, qui peut s'appuyer sur des techniques linguistiques variées ». Dans le prolongement de leur réflexion, je parlerai du jeu de mots en tant qu'acte de discours volontaire qui, tout en visant l'amusement, entraîne une proposition de reconfiguration de la perception du monde chez le récepteur. Chez Vian les jeux de mots servent la distorsion du langage et permettent ainsi d'interroger certaines tournures langagières. Esme Winter-Froemel et Angela Zirker (2015b : 7-8) remarquaient cette particularité des jeux de mots qui « peuvent remettre en question de manière ludique le fonctionnement du langage et explorer ses limites de manière créative ». Ils participent ainsi de la création langagière.

La syllepse est fortement mise en lien avec les jeux de mots, notamment sur son versant négatif. Dumarsais dit ainsi d'elle qu'elle « joue trop sur les mots pour ne pas demander bien de la circonspection » ([1730] 1988 : 134). Mon corpus ne dément pas cette assimilation de la syllepse au jeu de mots puisque l'intégralité des syllepses relevées dans l'œuvre de Vian est au centre du jeu de mots. Cette étude permettra de comprendre le fonctionnement des jeux de mots s'appuyant sur des syllepses dans un corpus vianesque.

Nous verrons que la syllepse, si elle sert de support au jeu de mots dans l'œuvre de Boris Vian, ne peut être réduite au jeu de mots puisque sa valeur énonciative de cumul de points de vue en confrontation ${ }^{2}$ et sa pragmatique complexe en font un outil essentiel de la fictionnalisation. Elle permet de s'appuyer

1 Pour chacune de ces œuvres, l'édition retenue est celle de la Pléiade (2010).

2 Pour cette notion, voir Alain Rabatel (1998, 2003, 2008). 
sur un savoir commun (par son lien avec le défigement) afin de repenser ces formes, et de proposer une nouvelle représentation de la réalité au lecteur.

Je propose donc d'étudier la dimension énonciative et pragmatique de la syllepse chez Vian et son apport à la construction fictionnelle.

Je commencerai ainsi par étudier le lien entre la syllepse et le jeu de mots dans un corpus vianesque, puis je m'interrogerai sur l'hétérogénéité énonciative présente dans la figure, ce qui nous permettra d'ébaucher une pragmatique de la syllepse chez Vian.

\section{La syllepse comme jeu de mots}

La syllepse est une figure du discours. Elle est généralement définie comme une figure du signifié s’appuyant sur la polysémie. Georges Molinié précise d'ailleurs dans son Dictionnaire de rhétorique (1992 : 311) que « la syllepse [...] consiste en ce qu'un terme, dans un discours, apparaissant dans le segment une fois, est pris en plusieurs sens, deux au moins, l'un tropique et l'autre pas, ou les deux tropiques, mais différemment ». Alain Rabatel (2015) remet cependant en cause le critère de non-répétition du point nodal. La figure permet selon lui un rapprochement de deux isotopies autour d'un point nodal polysémique, celui-ci pouvant être répété ou non. La syllepse rassemble les différents sens d'un même terme. Ces sens sont textuellement activés en contexte et créent ainsi un croisement isotopique au niveau du point nodal. Catherine Rouayrenc (2006 : 157) voit dans la syllepse « un carrefour d'isotopies (je parlerai de point nodal), le lieu de convergence de plusieurs parcours interprétatifs, mais cette convergence, qui se traduit par l'actualisation de deux signifiés pour un même signifiant, peut-être réalisée de manière différente, selon la place des termes qui génèrent l'isotopie dans laquelle vient s'insérer la syllepse ».

Chez Boris Vian, on rencontre le plus fréquemment les syllepses dites «bilatérales » qui sont définies par Catherine Rouayrenc comme suit :

les deux isotopies actualisées dans le point nodal que constitue la syllepse sont définies, l'une par le cotexte gauche, l'autre par le cotexte droit. Le cotexte gauche détermine un parcours interprétatif qui induit l'actualisation d'un signifié, alors que le cotexte droit induit pour la même lexie l'actualisation d'un autre signifié. Dans ce cas, le cotexte droit, ne continuant pas l'isotopie précédente [...] détermine une nouvelle isotopie dont l'existence est liée ou amène une réinterprétation du signifié de la lexie précédente, qui se charge alors d'un nouveau signifié. (2006: 160) 
Le rapport au cotexte de la figure développe ou non un effet de surprise et une opacité interprétative. Cet élément, ainsi que le chemin interprétatif à parcourir pour le lecteur selon les cas de figure, signalent la figure comme créatrice de sens au sens fort. C'est ce qui explique la popularité de la figure dans les jeux de mots : elle permet un jeu sur le sémantisme des mots, tout en proposant une représentation inédite d'un référent.

\subsection{Syllepse contre calembour}

L'affinité de la syllepse avec le jeu de mots amène parfois à la voir rangée avec le calembour dans les cas de polysémie (Guiraud 1979; Baudin 1973). Dans les romans de Vian, syllepses et calembours cohabitent, avec, cependant, une grande prégnance de la syllepse. S’ils participent tous les deux du jeu de mots, ils correspondent chacun à un procédé bien précis.

(1) Chloé avait mis, pour se rendre chez le docteur, une petite robe bleu tendre, décolletée très bas en pointe et portait un manteau en lynx, accompagné d'une toque assortie. Des chaussures de serpent teint complétaient l'ensemble. $\left(\mathrm{EJ}^{3}\right.$ : 434)

Le calembour, selon Anna Jaubert (2011 : 40), est une «figure de mots à la vocation d'une figure de pensée, le calembour se manifeste par un énoncé bref, mais qui pointe vers une langue de complicité : il laisse entendre une connivence avec son récepteur, en le renvoyant à un réseau sous-jacent de savoir et d'opinions partagés ». De manière formelle, il s'appuie sur une relation homonymique plus ou moins proche entre deux termes ou locutions. En (1), le jeu s'appuie sur la similitude phonique entre « serpent teint » et "serpentins ». En contexte, la présence du serpent est complètement appropriée alors que les serpentins viennent rompre la cohérence textuelle. Le calembour sert les fins du jeu de mots puisqu'il participe de la création d'une connivence entre le lecteur et le narrateur, mais il manifeste cependant une certaine gratuité. La syllepse ancre textuellement les deux sens qu'elle développe.

(2) Les souliers jaunes frémirent nerveusement sur les pédales de la commande, et avec le bruit d'un coucou qui s'envole, la voiture démarra. On croyait même entendre le choc des poids du coucou sur les murs. (TA : 43)

3 Les titres des œuvres de Boris Vian sont abrégés en TA (Troubles dans les andains); VP (Vercoquin et le plancton); EJ (L'Écume des jours); AP (L'Automne à Pékin) ; HR (L'Herbe rouge). 
Cet extrait de Troubles dans les andains est assez complexe. Il permet d'illustrer « la syllepse in presentia » (Rabatel : 2015). La répétition du substantif coucou nie normalement la possibilité de la syllepse (puisque, traditionnellement, la figure a un point nodal à occurrence unique). La première occurrence de " coucou » peut être interprétée de deux manières : soit il renvoie à l'oiseau soit il renvoie à la désignation d'un avion. La deuxième interprétation est bloquée par le verbe s'envole puisqu'un avion ne s'envole pas mais décolle. Si le contexte mécanique laisse attendre le sens aéronautique, le verbe trompe cette attente et réoriente le sens de coucou vers l'animalier. La deuxième occurrence de coucou a le sens de " pendule ». Ce substantif est ainsi le lieu d'une syllepse puisque le terme est polysémique. La complexité de cet exemple dévoile bien le potentiel de la figure dans les jeux de mots : elle opacifie le référent, fait cohabiter les différents sens d'un terme, créant ainsi un référent chargé de ses différents signifiés, et ancre son fonctionnement dans le contexte. Je rejoins ainsi le point de vue de Catherine Kerbrat-Orecchioni qui considère la syllepse comme la reine du jeu de mots : elle a un rendement pragmatique et textuel complexe rendant toute sa pertinence au jeu de mots. ${ }^{4}$

\subsection{Syllepse et défigement}

Dans mon corpus, la syllepse a un lien privilégié avec le défigement, puisque deux tiers des syllepses relevées touchent une forme figée. ${ }^{5}$ Selon Béatrice Lamiroy et Jean-René Klein (2016 : 15), une séquence figée se définit comme « une séquence polylexicale préfabriquée qui, ne procédant pas d'une combinatoire sémantico-syntaxique libre, fait partie de la compétence lexicale d'un locuteur. [Collocations, expressions figées, phrases situationnelles et proverbes] constituent des séquences préfabriquées, inscrites dans la mémoire du locuteur natif ». Ainsi, une variation syntaxique, sémantique ou contextuelle entraînera un défigement de la séquence figée en proposant une réévaluation du sens de la séquence figée. Chez Boris Vian, la syllepse est outil de la déconventionnalisation de la langue et joue ainsi avec le défigement dans le but de tromper les attentes (ou habitudes) du lecteur.

4 Catherine Kerbrat-Orecchioni, lors de sa communication « Heurs et malheurs du jeu de mots ", dans le cadre du colloque «Dynamique du jeu de mots, perspectives interdisciplinaires », qui s'est tenu du 29 septembre au 1er octobre 2016 à Trier (voir Kerbrat-Orecchioni, ce volume).

5 Pour plus de précisions sur le fonctionnement de la figure lorsqu'elle touche une forme figée voir Alain Rabatel (à paraître) qui étudie le lien entre syllepse, antanaclase et formes figées. 
Le défigement intervient lorsqu'une locution figée et en partie dé-sémantisée est réactivée en contexte, en général par remotivation sémantique. La syllepse permet de repenser le sens de l'expression et de faire fusionner le sens « habituel » de l'expression avec le sens original créé par la figure.

(3) Le Maire toussota dans son cornet acoustique et prit la parole par le cou pour l'étrangler, mais elle tint bon.

« Messiers, dit-il, et cher coadjupiles. » (HR : 322)

(4) Emmanuel avait tellement peigné la girafe ce matin-là que la pauvre bête en était morte. Des touffes de ses poils traînaient un peu partout, et son cadavre, dont on avait fait passer la tête par la fenêtre, pour pouvoir circuler, gisait sous le bureau d'Adolphe Troude, qu'encombraient déjà quatre tonnes d'engrais divers, logés dans des petits sacs de toile, car cet estimable individu s'adonnait à la culture maraîchère dans son jardin de Clamart. (VP : 174)

Ces deux exemples touchent une forme figée. En (3), la syllepse touche la collocation prendre la parole, qui signifie « commencer à parler » ou « tirer la parole vers soi ». Le point nodal de la figure se situe au niveau du verbe « prendre » qui a, dans la collocation, un sens abstrait. Dans le cadre du jeu avec le figement, la syllepse ne met pas seulement en commun les différents sens du verbe, elle fait aussi cohabiter le sens de la collocation avec celui du collocatif. Cet exemple permet de remarquer la structure du jeu de mots dans une collocation lorsque celui-ci est porté par une syllepse. En désolidarisant deux éléments formant un tout, le texte oblige le lecteur à repenser au sens de cette collocation non-compositionnelle. Par la syllepse, le narrateur joue des sens activés : au lieu d'activer le sens concret du verbe (caractéristique souvent usitée dans la définition de la figure), la syllepse fait advenir un sens complètement inattendu dans le cotexte droit. Ce type de jeu de mots est assez facile, et pourrait être assimilé à une prise au pied de la lettre (si c'était le sens concret du verbe qui était développé textuellement). Il permet néanmoins d'apporter de l'humour et de l'étrangeté dans l'œuvre.

L'exemple (4) est généralement perçu (Lapprand 1988 ; Baudin 1973 ; Weiss 2014) comme une prise au pied de la lettre d'expression figée. Or, il s'agit plutôt ici d'une syllepse touchant l'expression figée peigner la girafe, signifiant « ne rien faire ». Le sens abstrait de l'expression est ici mêlé à son sens compositionnel. Alors que le contexte renvoie directement au sens abstrait de l'expression, le cotexte droit renvoie au sens concret puisqu'il mentionne la présence de l'animal. Selon Catherine Rouayrenc (2006 : 165), cette syllepse bilatérale « crée évidemment un effet de surprise puisque le parcours interprétatif amorcé du fait du cotexte gauche est interrompu et remis en question par le cotexte droit [...]. Cette 
syllepse, qui exige réinterprétation, est déception ». Elle est déception puisqu'elle contredit le sens amorcé par le cotexte gauche et va de ce fait contre les attentes du lecteur. Ce type d'occurrence est particulièrement fonctionnel dans les jeux de mots puisqu'il garantit un effet de surprise, compagnon habituel du ludique.

Lorsque la syllepse défige une expression, elle ajoute au jeu une déstabilisation du lecteur. La figure entre alors dans un mouvement de déconventionnalisation de la langue.

\subsection{Une langue déconventionnalisée}

En effet, la syllepse entraîne une reconfiguration des formes normées du langage. Vian dépiste les images présentes dans le langage afin de les détourner de leur emploi habituel. Si on a pu voir l'affinité de la syllepse dans les formes figées, elle entre, de manière générale, dans un mouvement de détournement des tournures langagières.

(5) Le froid retenait les gens chez eux. Ceux qui réussissaient à s'arracher à sa prise y laissaient des lambeaux de vêtements et mouraient d'angine. (EJ : 368)

(6) Il se remit debout et jura sans ostentation ; un assez gros mot, mais bien proportionné ; puis il suivit olive, mais sans se presser, parce qu'elles exagèrent. (AP : 581-582)

L'extrait en (5) s'appuie sur la personnification du froid qui prend ainsi en otage les habitants. La syllepse tient de la cohabitation d'un sens concret - les températures basses empêchent les gens de sortir - et d'un sens abstrait et métaphorique - le froid prend les individus en otage. Le froid est, dans l'acception courante, la cause de l'empêchement ; par la syllepse, il devient sujet du verbe et est promu au rang d'entité fictionnelle agissante. Le lecteur est alors en présence d'un monde où les entités météorologiques ont une force agissante. Il ne s'agit pas ici de merveilleux, mais d'un remodelage de la réalité par le biais d'un point de vue déplacé : ce n'est pas une représentation doxique du monde, mais une représentation subjective en lien avec les sensations. Le froid devient un agent dans la fiction, une sorte de personnage qui possède des attributs.

Le jeu de mots ici vient du renversement de ce que Lakoff et Johnson (1986) appellent une métaphore conceptuelle. Le langage concernant le rapport de l'homme aux intempéries est construit sur une métaphore de la lutte. Cette métaphore structurante est détournée par la mise en œuvre d'une lutte réelle entre l'homme et la nature. 
L'exemple (6) illustre bien l'impact de la syllepse sur des formes conventionnelles. Le syntagme gros mot est un mot composé, il se situe donc entre la forme figée et le substantif. Les "gros mots » regroupent tous les termes jugés vulgaires. L'adverbe assez exprime l'intensité moyenne de la propriété d'ordre quantitatif de la grosseur du mot. La syllepse porte sur l'adjectif gros (ce qui perturbe la coalescence du nom composé), son identification amorcée avec assez est pleinement entérinée dans le contexte droit par l'ajout en incidente de, mais bien proportionné, qui sert à rectifier la portée de la caractérisation précédente. Le jeu de mots crée un nouveau référent fictionnel, le «mot de grande taille» dont on peut évaluer l'aspect. Quoiqu'incongrue, cette nouvelle acception est soumise à la sagacité du lecteur, qui peut s'arrêter au sourire en pointant le manque d'adéquation au « réel » de ce « gros mot », ou peut entériner ce nouveau référent fictionnel.

Le détournement langagier est au cœur de ces jeux, mais l'on peut déceler autre chose : la construction d'un monde où le principe est la dé-régularisation. Les romans de Vian sont saturés de ce langage détourné, au point que les jeux langagiers érigent une norme de lecture et des attentes très fortes du lecteur. Audelà du jeu avec le défigement et les dé-conventionnalisations qui renvoient à un processus scriptural quasi iconoclaste, les syllepses dévoilent un processus créateur global, caractérisé par une forte hétérogénéité énonciative, à l'origine d'une vision décalée d'un même référent.

\section{L'hétérogénéité énonciative dans la syllepse}

L'hétérogénéité énonciative se caractérise par un doute sur l'unicité du sujet parlant. La syllepse est un lieu idéal pour la confrontation de différentes voix par la confusion isotopique qu'elle instaure. La figure devient alors la représentante de ce conflit énonciatif sur un même objet. Jacqueline Authier-Revuz précise le lien entre polysémie et hétérogénéité énonciative. Selon elle (1984 : 104), « une autre modalité de prise de sens pour un mot, recourant explicitement à l'ailleurs d'un autre discours spécifié ou à celui de la langue comme lieu de polysémie» constitue un point d'hétérogénéité. L'approche énonciative permettra de montrer que la syllepse englobe les réalités subjectives des différents sujets afin d'interroger les constructions langagières et de souligner l'importance des mots choisis. 


\subsection{L'articulation des représentations sur le monde dans la syllepse}

Puisque la syllepse développe deux isotopies différentes, il paraît normal de se demander qui prend en charge les deux sens mis en place par la syllepse. La figure présente une confrontation de points de vue. Le point de vue correspond, selon Alain Rabatel (2005 : 56) «à un contenu propositionnel renvoyant à un énonciateur auquel le locuteur «s'assimilè ou au contraire dont il se distancie ». Ce mélange des points de vue offre une représentation complexe du référent.

Dans l'exemple suivant, Colin se rend dans les vestiaires de la piscine Molitor.

(7) Un homme à chandail blanc lui [Colin] ouvrit une cabine, encaissa le pourboire qui lui servirait pour manger, car il avait l'air d'un menteur, et l'abandonna dans cet in-pace après avoir, d'une craie négligente, tracé les initiales du client sur un rectangle noirci disposé, à cet effet, à l'intérieur de la cabine. (EJ : 355-356)

L'exemple (7) signale de façon très claire la confrontation des points de vue. La syllepse trouve son point nodal dans le nom composé par soudure totale «pourboire ». Il désigne couramment une somme d'argent donnée en plus de la tarification normale, comme récompense d'un travail ou d'un service. Considérant le cotexte droit (le circonstant de but pour manger), le sens de pourboire fait l'objet d'une interprétation étroite : celui-ci est destiné à boire et non à manger. Ainsi l'extrait suivant : il « encaissa le pourboire qui lui servirait pour manger, car il avait l'air d'un menteur » fait collaborer deux logiques de pensée. La première, la plus courante, est celle de l'homme à chandail blanc qui considère le pourboire comme une rétribution pouvant être utilisée à sa discrétion. La seconde - littéralisante - correspond à celle de Colin qui restreint le pourboire à ce pour quoi il est dit être fait, se posant en en littéraliste et en «puriste » de la langue. La confrontation des points de vue est signalée dans l'extrait par l'emploi du conditionnel présent servirait qui est susceptible de deux interprétations : soit le conditionnel est un futur du passé, dans la dépendance du verbe au passé simple (allait lui servir), et appartient à la narration, soit la relative explicative est imputable à Colin qui imagine l'utilisation de ce pourboire par l'homme au chandail blanc, comme l'autorise à penser la justification de l'énonciation de cette relative inaugurée par car.

La syllepse manifeste deux points de vue opposés sur le même objet - le pourboire - et entraîne un quiproquo entre les personnages. Cette confrontation orchestrée par le narrateur se poursuit sur un mode ludique dans un ultime jeu de mots sur in pace. L'« in pace », qui désigne ici la « cabine », lieu retiré, secret, 
est aussi une «impasse " si on prononce le mot à la française. Le texte laisse affleurer la présence d'un narrateur qui met en présence des points de vue hétérogènes au service d'une fécondité interprétative. La confrontation des points de vue n'est pas résolue par le narrateur. Chaque représentation subjective de la réalité persiste. Il y a ici une impasse de la syllepse complètement assumée par le narrateur (visible dans le jeu sur in pace/impasse).

\subsection{L'interaction des discours dans la syllepse}

Le développement entraîné par la figure est marqué par un dialogisme fort. Ce dernier peut être défini comme « la capacité de l'énoncé à faire entendre, outre la voix du locuteur-énonciateur, une (ou plusieurs) autre(s) voix qui le feuillete(nt) énonciativement » (Bres 2007 : 38). Dans les exemples que nous allons étudier, le dialogisme laisse entendre un narrateur guidant le lecteur dans l'œuvre. Le dialogisme devient alors un lieu d'interaction du narrateur avec son lecteur.

(8) Les pas de Jacquemort résonnèrent dans l'escalier. En même temps, les cris de la femme cessèrent et Angel resta frappé de stupeur. S'approchant doucement de la porte, il essaya de voir, mais le pied du lit lui masquait tout le reste et il se tordit douloureusement l'œil droit sans résultat appréciable. Il se redressa et tendit l'oreille, à personne en particulier. $(\mathrm{AC}: 505)$

Dans l'extrait (8) la syllepse est localisée dans le verbe tendre: associée à « l'oreille » il signifie « écouter attentivement » et en emploi autonome " présenter un objet à quelqu'un », le groupe nominal « l'oreille » étant dans le second cas un complément libre. Cet exemple semble plus facile, et plus gratuit que les autres. Cette gratuité du jeu de mots se perçoit par le peu d'ancrage contextuel de la seconde isotopie : le sens de « passer son oreille à quelqu'un » n'est justifié que par la présence du verbe tendre.

Deux énonciations sont ici mêlées (ce qui est marqué par le détachement par la virgule). L'autonomie énonciative du groupe prépositionnel détaché signale une deuxième énonciation de type humoristique. L'ajout hyperbatique «à personne en particulier ", consiste en une énonciation détachée surnuméraire qui vient préciser quelque chose qui n'en a pas besoin (d'où la gratuité). Il s'agit ici d'autodialogisme où le narrateur précise de manière superflue son propre discours. $^{6}$

6 L'autodialogisme correspond à un discours du locuteur énonciateur avec les « autres de soi » qui « correspondent aux diverses positions énonciatives que E1 adopte lorsqu'il envisage les 
L'extrait (9) est marqué par le dialogisme interlocutif anticipatif. Ce type de dialogisme « peut être analysé comme réponse anticipée à la réponse potentielle que le locuteur prête à l'allocutaire » (Bres et Nowaskowska 2008 : 25). L'intérêt de ce type de dialogisme est qu'il explicite à la surface du texte la relation entre narrateur et lecteur, l'un ne cessant d'anticiper sur la compréhension de l'autre, le second ne cessant de se faire proposer une certaine lecture du texte.

(9) « Je veux penser à autre chose... Supposons que je me casse la gueule dans l'escalier... » Le tapis de l'escalier, mauve très clair, n'était usé que toutes les trois marches : en effet, Colin descendait toujours quatre à quatre. Il se prit les pieds dans une tringle nickelée et se mélangea à la rampe. (EJ : 367)

Cet exemple comporte une syllepse autour de l'expression quatre à quatre, et de l'explication de l'usure du tapis. Elle associe deux emplois proches de quatre à quatre : un emploi «concret » (à l'origine de l'expression) signifiant descendre ou monter un escalier plusieurs marches à la fois, et un emploi dérivé du premier, au sens de «très rapidement ». Ayant signalé l'usure du tapis à intervalle régulier, le narrateur anticipe sur la question que le lecteur pourrait se poser et lui fournit la réponse par le biais de la syllepse. La figure sert donc ici à normaliser un fait fictionnel qui pourrait paraître étrange au lecteur.

Par le biais du dialogisme interlocutif anticipatif, le narrateur prévient un défaut de compréhension et donne ainsi un surplus de sens à même de pointer la syllepse comme lieu de création de la norme fictionnelle. Ce type de dialogisme paraît alors particulièrement efficace dans le cadre de la fictionnalisation puisqu'il permet au narrateur de négocier la fiction (comme on négocie un virage) tout en jouant sur les normes langagières du lecteur. Cette attitude narratoriale est caractéristique de l'univers romanesque de Vian, et se joue ad libitum dans son œuvre.

De ce fait, ce qui paraît être intuitivement pour le lecteur un jeu de mots ne l'est pas obligatoirement. Par l'hétérogénéité énonciative présente autour des syllepses, celles-ci mettent en place un lieu de rencontre de différents points de vue sur un même objet. Si le résultat fait rire, les points de vue évoqués, eux, ne visent pas forcément le jeu (comme en (7)). Il faut donc aller au-delà de l'effet jeu de mots de la syllepse pour en percevoir la visée première, centrée sur la construction fictionnelle d'un référent.

choses de tel ou tel point de vue, dans tel ou tel cadre spatiotemporel, tel ou tel cadre de référence : ainsi l'énonciateur dialogue avec des ‘autres` de lui-même, codés (L1'/)E1' » (Rabatel $2012: 30)$. 


\section{La pragmatique de la syllepse : normalisation et négociation de la fiction}

La confrontation des points de vue ainsi que le dialogisme permettent de donner un aperçu de la logique fictionnelle mise en place par la syllepse. La figure, liée certes souvent au jeu de mots, ne s'y réduit pas et entraîne une reconfiguration de la réalité. L'effort que déploie le texte pour normaliser la fiction est soutenu localement par les syllepses.

\subsection{Une normalisation contextuelle de la syllepse}

Le développement accompagnant la syllepse permet d'ancrer la figure dans une normalité fictionnelle. Il réduit alors le caractère étrange ou inouï de ce qui est évoqué et modifie la réception : il atténue l'incrédulité du lecteur et le porte à adhérer non seulement au monde représenté, mais à ses règles et à sa logique. Pour autant, le monde vianesque n'est ni fantastique : il ne fait pas naître d'inquiétude, ni merveilleux : il n'est pas transporté hors du monde réel de référence du lecteur.

(10) Il n'y a qu'une chose gênante, dit Colin, c'est la pédale forte pour l'œuf battu. J'ai dû mettre un système d'enclenchement spécial, parce que lorsqu'on joue un morceau trop « hot », il tombe des morceaux d'omelette dans le cocktail, et c'est dur à avaler. Je modifierai ça. Actuellement, il suffit de faire attention. (EJ : 353)

Dans cet extrait, le jeu de mots se fait autour de l'adjectif anglais « hot ». Il est le point nodal de la syllepse. En anglais, cet adjectif se traduit par «chaud». Il existe également un autre emploi de « hot » dans le langage populaire, lorsqu'il est utilisé pour désigner un tempo rapide. Dans le premier cas, on pourrait envisager un jeu simple sur la polysémie de l'adjectif tirant profit surtout du sens commun, l'œuf battu chauffé donnant une omelette, et ce pour les fins du jeu de mots. Dans le second cas, la polysémie est rendue pertinente en contexte puisque la pédale peut effectivement participer à la création d'une omelette si l'on joue un morceau trop rapide. Le mouvement rapide de la pédale s'apparente alors à celui du fouet permettant le durcissement de l'œuf. Ce point de vue n'est pas tant loufoque qu'hyperbolique. Ainsi, les deux signifiés engendrés par la syllepse sont mêlés. L'entremêlement des deux sens est confirmé en contexte par « c'est dur à avaler » qui normalise l'imagerie véhiculée par la figure. On voit bien alors que la figure ne saurait se réduire au jeu de mots, mais qu'elle est un véritable moteur de la fiction. 
(11) Le mobilier de cette pièce comprenait en outre un long meuble bas, aménagé en discothèque, un pick-up du plus fort module et un meuble, symétrique du premier, contenant les lance-pierres, les assiettes, les verres et les autres ustensiles que l'on utilise chez les civilisés pour manger. (EJ : 350)

En (11), la syllepse prend son appui sur l'expression manger avec un / à coup de lance-pierre (qui est implicite et inférable) signifiant manger très rapidement et de manière insuffisante. Le terme « lance-pierres » est alors chargé d'un double sens : un, abstrait, exprimant la rapidité et l'autre, concret, renvoyant à une arme permettant d'envoyer des pierres, ou autres projectiles, grâce à un élastique attaché à une base en forme d'Y. La figure, en prenant le sens concret, fait advenir des lance-pierres dans la vaisselle courante. Le repérage de la syllepse permet de normaliser la présence de cet ustensile dans le vaisselier. La contradiction entre «les civilisés » et leur utilisation des «lance-pierres » souligne la critique ironique sous-jacente. La syllepse renforce l'idée d'une société qui ne prend plus le temps ni de manger, ni de bien manger. Ainsi, si la première lecture peut être dérangeante par l'incongruité de la présence des lance-pierres au début de l'énumération de la vaisselle présente dans le meuble, le ton ironique de la fin du paragraphe résout cette incohérence en montrant que l'expression est devenue réalité et que l'objet lance-pierre fait partie des habitudes de vie.

On remarque donc bien chez Vian que notre réalité est confrontée à une réalité autre où l'abstrait a une concrétude mise au service de la fiction : c'est ce que fait la syllepse chez Vian, elle opère le chemin inverse : non pas du sens concret vers le sens abstrait - sens de la dérivation sémantique traditionnelle (du lance-pierre à la rapidité) -, mais du sens abstrait vers un nouveau sens concret. Elle convertit l'abstrait en un concret renouvelé au service d'une fiction qui ne se résume pas à de l'invention potache ou spirituelle. Il parait ainsi évident que la syllepse engendre une rencontre fructueuse entre deux mondes, deux univers de pensée, et la création d'un troisième hybride.

\subsection{La syllepse comme norme de lecture}

Cette normalisation de la figure par le contexte permet de comprendre que la figure n'est plus un écart, mais l'unique moyen de désigner le référent en question. Elle est ainsi pleinement créatrice de sens.

(12) « Une de vos machines a l'air de s'emballer... dit Colin en désignant l'engin en question.

- Ah !... » dit le marchand de remèdes.

Il se pencha, prit sous son comptoir une carabine, épaula tranquillement et tira. La machine cabriola en l'air, et retomba pantelante. 
« Ce n'est rien, dit le marchand. De temps en temps le lapin l'emporte sur l'acier et il faut les supprimer.»

Il souleva la machine, appuya sur le carter inférieur pour la faire pisser et la pendit à un clou. (EJ : 430)

Il s'agit ici d'une syllepse entraînant un jeu par le détournement de l'emploi du verbe s'emballer pour les machines. Deux isotopies sont présentes : celle animalière et celle de la mécanique. Ces deux domaines se croisent par le verbe s'emballer.

Si l'on peut penser que la figure animalise la machine et entraîne ainsi un développement incongru, ce n'est pas le cas. Une fois le jeu de mots perçu, on peut se rendre compte de sa légitimité en contexte. En effet, comme le signal Chick en amont de l'extrait, la machine est « un lapin modifié ». Il explique qu' " on conserve la fonction qu'on veut. Là [le marchand] a gardé les mouvements du tube digestif, sans la partie chimique de la digestion. C'est bien plus simple que de faire des pilules avec un pisteur normal» (EJ : 428). Ainsi la syllepse est justifiée par la nature de la machine. Les deux isotopies se fondent afin d'être représentatives de l'objet à décrire. Le texte crée alors ses propres normes que le lecteur doit accepter pour réussir son immersion fictionnelle.

La syllepse engendre la création d'un univers fictionnel décalé et hybride (et non seulement contrefactuel) en faisant le chemin de l'abstrait vers le concret issu de la rencontre de deux univers, dont l'un ressemble à notre monde. Elle normalise cet univers fictionnel auprès du lecteur et donc le négocie auprès de lui (ce pour quoi il y a du dialogisme interlocutif anticipatif) afin qu'il y adhère activement.

\section{Conclusion et ouverture : aux risques de la syllepse}

La syllepse ne se laisse pas enfermer dans cette «normalisation » d'une fiction débridée et représente donc un "risque » : celui pour le lecteur de se laisser aller dans une routine de lecture, et de repérer trop automatiquement les syllepses. Dans l'exemple (13), le narrateur en fait voir une pour se raviser après :

(13) Le soleil tapait dur sur les dalles du port qui s'enfonçaient peu à peu... C'était peut-être seulement la marée qui montait. (TA : 92)

Dans cette occurrence, la syllepse n'entraîne pas une personnification du soleil, mais bien un jeu de double sens sur le verbe taper. L'expression « le soleil tapait » 
signale la forte chaleur émise par les rayons du soleil. Le second sens développé par la syllepse est l'expérience concrète du verbe « taper ». De cette manière, le second sens bouleverse le sens prototypique de l'expression et laisse percevoir une nouvelle réalité où la force du soleil se perçoit de manière concrète. La suite de l'extrait remet en question la syllepse et introduit un autre point de vue, celui d'un narrateur cherchant une explication conforme aux normes du monde de référence du lecteur : la lecture de la syllepse et son interprétation sont alors bloquées par « C'était peut-être seulement la marée qui montait ». Le lecteur, au moment où il se glissait dans les normes fictionnelles construites par la syllepse, est vite " ramené à la réalité », celle de la marée ; la figure était cette fois-ci une fausse piste : elle n'évoquait pas une norme différente, mais une simple vision décalée de la réalité. Ici, l'auteur affleure dans ce jeu de contre-pied avec son lecteur.

La syllepse, dans le jeu de mots, met en place une stratégie de restructuration du langage en entraînant une représentation du monde autre que celle que nous connaissons. En ce sens, la syllepse peut à première vue renvoyer à un jeu de mots, mais si l'on rentre dans la logique intrafictionnelle, elle n'est qu'un moyen de représenter les normes fictionnelles. Elle donne ainsi la clé d'interprétation de l'œuvre de Boris Vian : cette dernière n'est pas fantastique, elle représente la réalité à travers le prisme de la perception de l'auteur lui-même et de son travail sur la langue. ${ }^{7}$

La syllepse est alors une figure efficace de la fictionnalisation dans le sens où elle devient outil de la création d'un monde : le cotexte gauche rend hommage à la norme alors que le cotexte droit représente ce décalage entre le monde du lecteur et celui de la fiction. Elle sert la création d'un monde ni factuel, ni contrefactuel, mais para-factuel.

Par conséquent, le jeu de mots qui s'appuie sur la syllepse, chez Vian, n'est que très rarement gratuit : il engendre la création d'un monde rendant compte de la complexité du langage. La figure, dans le jeu de mots, possède une double visée pragmatique : elle participe du construit fictionnel (à la fois en prise et dérogeant de l'univers de référence) et elle noue une relation très particulière avec le lecteur.

7 Jacques Bens évoque d'ailleurs un « langage-univers» (1963) pour décrire de L'Écume des jours. Plus tard, il revient sur la particularité de Vian en avançant que « ce qu'il y a de nouveau dans l'approche de Boris Vian (j'allais dire : «dans sa méthode`, mais il est bien question de méthode !), ce qui fait de son écriture un outil très personnel, c'est qu'il utilise le langage à l'état brut, comme un matériau en quelque sorte naïf. Loin de fabriquer des panoplies inédites, en plaçant côte à côte des mots jusque-là peu faits pour se rencontrer, il découvre, et nous fait découvrir, des objets insolites au milieu d'un appareil verbal qui nous est familier » (1976:130). 


\section{Références bibliographiques}

Authier-Revuz, Jacqueline. 1984. Hétérogénéité énonciative. Langages 73. 98-111.

Baudin, Henri. 1973. Boris Vian, humoriste. Grenoble : Presses Universitaires de Grenoble.

Bens, Jacques. 1963. Un langage univers. Postface de L'Écume des jours (Boris Vian). Paris : Gallimard.

Bens, Jacques. 1976. Boris Vian. Paris : Bordas.

Bres, Jacques. 2007. Sous la surface textuelle, la profondeur énonciative. Les formes du dialogisme de l'énoncé. In Rita Therkelsen, Møller Andersen, Nina, Nølke, Henning (éds.), Sproglog Polyfoni, 37-54. Aarhus : Universitetsforlag.

Bres, Jacques \& Aleksandra Nowakowska. 2008. « J'exagère ?... » Du dialogisme interlocutif. In Merete Birkelund, Maj-Britt Mosegaard Hansen \& Coco Norén (éds.), L'Énonciation dans tous ses états, 1-27. Berne : Peter Lang.

Dumarsais, César Chesneau. [1730] 1988. Des Tropes ou des différents sens ou Des différents sens dans lesquels on peut prendre un mot dans une même langue. Paris : Flammarion.

Guiraud, Pierre. 1979. Les Jeux de mots. Paris : Presses Universitaires de France.

Jaubert, Anna. 2011. Le calembour ou la pragmatique du trait /facile/. Le Français moderne 79(1). 33-43.

Lakoff, Georges \& Mark Johnson. 1986. Metaphors we live by [Les Métaphores de la vie quotidienne]. Paris : Éditions de Minuit.

Lamiroy, Béatrice \& Jean René Klein. 2016. Le figement : unité et diversité, collocations, expressions figées, phrases situationnelles, proverbes. L'Information grammaticale 148. 15-20.

Lapprand, Marc. 1988. Vercoquin et le plancton comme pré-texte de L'Écume des jours. Naissance de l'écrivain chez Boris Vian. Texte 7. 249-266.

Lecolle, Michelle. 2015. Jeux de mots et motivation : une approche du sentiment linguistique. In Esme Winter-Froemel \& Angelika Zirker (éds.), Enjeux du jeu de mots. Perspectives linguistiques et littéraires (The Dynamics of Wordplay 2), 217-244. Berlin \& Boston : De Gruyter.

Molinié, Georges. 1992. Dictionnaire de rhétorique. Paris : Librairie Générale Française.

Rabatel, Alain. 1998. La Construction textuelle du point de vue. Lausanne : Delachaux \& Niestlé.

Rabatel, Alain. 2003. Présentation : Le point de vue, entre langue et discours, description et interprétation : état de l'art et perspectives. Cahiers de praxématique 41. 7-24.

Rabatel, Alain. 2005. Le point de vue, une catégorie transversale. Le Français aujourd'hui 151. 57-68.

Rabatel, Alain. 2008. Figures et points de vue en confrontation. Langue française 160. 3-19.

Rabatel, Alain. 2012. Positions, positionnements et postures de l'énonciateurs. Revue Tranel 56. 23-42.

Rabatel, Alain. 2015. La plurisémie dans les syllepses et les antanaclases. Vox Romanica 74. 124-156.

Rabatel, Alain. À paraître. La créativité verbale dans les devinettes : points de vue cumulatifs, assertions non sérieuses et sous-énonciation. À paraître dans : Bettina Full \& Michelle Lecolle (éds.), Jeux de mots et créativité. Langue(s), discours et littérature (The Dynamics of Wordplay 4). Berlin \& Boston : De Gruyter. 
Rouayrenc, Catherine. 2006. Syllepse et co(n)texte. In Henri Chevalier \& Philippe Wahl (éds.), La syllepse, figure stylistique, 157-172. Lyon : Presses Universitaires de Lyon.

Véron, Laélia. 2015. Jeu de mots et double communication dans l'œuvre littéraire : l'exemple de La Comédie humaine de Balzac. In Esme Winter-Froemel \& Angelika Zirker (éds.), Enjeux du jeu de mots. Perspectives linguistiques et littéraires (The Dynamics of Wordplay 2), 93114. Berlin \& Boston : De Gruyter.

Vian, Boris. 2010. CEuvres romanesques complètes. Paris : Gallimard.

Weiss, Martin. [1983] 2014. Boris Vian - la langue qui trébuche, jeux de mots dans l'œuvre d'un génie (Grazer Linguistische Studien 20), University of Graz, nouvelle édition (eBook).

Winter-Froemel Esme \& Angelika Zirker (éds.). 2015a. Enjeux du jeu de mots. Perspectives linguistiques et littéraires (The Dynamics of Wordplay 2). Berlin \& Boston : De Gruyter.

Winter-Froemel Esme \& Angelika Zirker. 2015b. Jeux de mots, enjeux et interfaces dans l'interaction locuteur-auditeur : réflexions introductives. In Esme Winter-Froemel \& Angelika Zirker (éds.), Enjeux du jeu de mots. Perspectives linguistiques et littéraires (The Dynamics of Wordplay 2), 1-27. Berlin \& Boston : De Gruyter.

Zirker Angelika \& Esme Winter-Froemel (éds.). 2015. Wordplay and Metalinguistic / Metadiscursive Reflection. Authors, Contexts, Techniques, and Meta-Reflection (The Dynamics of Wordplay 1). Berlin \& Boston : De Gruyter. 
